\title{
Eating patterns of Australian adults: associations with blood pressure and hypertension prevalence
}

\author{
Rebecca M. Leech ${ }^{1}$ (1) Anna Timperio ${ }^{1} \cdot$ Anthony Worsley $^{1} \cdot$ Sarah A. McNaughton ${ }^{1}$
}

Received: 23 March 2018 / Accepted: 5 June 2018 / Published online: 6 June 2018

(c) The Author(s) 2018

\begin{abstract}
Purpose Eating patterns have been linked to obesity, an established risk factor for hypertension; however, their contribution to hypertension is poorly understood. This study aimed to examine associations of frequency of meals, snacks and all eating occasions (EO), and temporal eating patterns, with blood pressure (BP) and hypertension.

Methods Dietary data collected via two 24-h recalls during the 2011-2012 Australian National Nutrition and Physical Activity Survey ( $n=4482$ adults, $\geq 19$ years) were analysed. Frequencies of EO, meals, and snacks were calculated. Temporal eating patterns were determined using latent class analysis. Multivariate regression models assessed associations of eating patterns with systolic BP (SBP), diastolic BP (DBP), and hypertension prevalence.

Results Among men, a higher snack frequency was inversely associated with DBP $[\beta=-0.59,95 \%$ confidence interval (CI) $(-1.12,-0.07)]$ and hypertension [odds ratio (OR) $0.86,95 \% \mathrm{CI}(0.75,0.98)]$ after adjustment for covariates and BMI. However, these associations disappeared after additional adjustment for total energy intake and overall diet quality. Among women, a temporal eating pattern characterized by a later "lunch" meal was associated with SBP $[\beta=2.45,95 \% \mathrm{CI}$ $(0.05,4.84)]$, DBP $[\beta=1.69,95 \% \mathrm{CI}(0.25,3.13)]$, and hypertension $[\mathrm{OR}=1.49,95 \% \mathrm{CI}(1.00,2.22)]$, when compared to a "conventional" eating pattern.

Conclusions In this study, an inverse association found between snack frequency and BP among men disappeared after adjustment for dietary factors and a "later lunch" pattern was associated with higher BP in women. Future research is needed to understand the relationship and potential mechanistic pathways between eating patterns and BP.
\end{abstract}

Keywords Blood pressure $\cdot$ Circadian rhythms $\cdot$ Eating frequency $\cdot$ Eating patterns $\cdot$ Meals $\cdot$ Meal timing $\cdot$ Snacks

\section{Introduction}

Poor dietary habits, obesity, and high systolic blood pressure (SBP) are three major risk factors contributing to the global burden of disease [1]. As these risk factors are strongly interrelated, it has been postulated that diet is a key driver of global increases in BMI and subsequent SBP [1]. The adoption of a dietary pattern rich in plant-based foods but low in red meat and discretionary (e.g., non-core)

Electronic supplementary material The online version of this article (https://doi.org/10.1007/s00394-018-1741-y) contains supplementary material, which is available to authorized users.

Rebecca M. Leech

rebecca.leech@deakin.edu.au

1 Institute for Physical Activity and Nutrition (IPAN), School of Exercise and Nutrition Sciences, Deakin University, 75 Pigdons Rd, Waurn Ponds, Geelong 3216, Australia foods has been identified as an important strategy for the prevention of cardiovascular disease and hypertension [2, 3]. However, the contribution of eating patterns to hypertension is poorly understood [4]. Eating patterns, including the frequency and temporality of eating occasions [(EO), e.g., meals and snacks], may represent a unique cardiometabolic risk factor [5]. This is because the metabolic and physiological functions in responses to food are also, in part, regulated by circadian clock systems. Thus, variations in the temporal distribution of food consumption may have important health implications [6].

Epidemiologic evidence [7-11] suggests that skipping breakfast and irregular meal habits are associated with poorer cardiometabolic health in adults, including incident hypertension [7]. However, evidence for associations with EO frequency is less consistent [4]. In three studies, a higher EO frequency (e.g., $\geq 5$ EO per day) was associated with lower SBP $[12,13]$ and diastolic blood pressure (DBP) [13, 
14]. In support of these findings, a review of short-term experimental studies found that six or more meals per day, compared to one meal, resulted in favourable decreases in cardiometabolic risk markers [15]. However, most of the reviewed studies did not control for differences in dietary intake and other population-based studies have found no evidence for a relation of $\mathrm{EO}$ frequency with blood pressure (BP) $[16,17]$. Furthermore, research suggests that meals and snacks are differentially related to dietary intakes [18, 19] and obesity $[19,20]$, yet studies examining meals and snacks as separate EO in relation to hypertension are rare [12].

The temporal distribution of EO in relation to BP has rarely been examined [21, 22]. In the UK Cohort Study, Almoosawi et al. [21] found a positive association for higher total energy intake in the evening with 10 -year increases in SBP and DBP. In a study of Spanish adult volunteers, consumption of an afternoon meal was associated with lower SBP and DBP [22]. However, these studies focussed on timing of EO/energy intake at isolated periods of the day and did not simultaneously capture EO at other times of the day [4]. Data-driven statistical techniques (e.g., cluster and latent class analysis) have recently been identified as useful tools for capturing temporal eating patterns across the day, but have only been used to examine relations with diet quality and obesity [23, 24]. Therefore, the aim of this study was to examine the associations of frequency of meals, snacks and all EO, and temporal eating patterns, determined using a latent class analysis approach, with BP and hypertension, in Australian men and women. Based on the findings of our earlier studies that found a positive relation for snacking frequency and a "grazing" temporal eating pattern with measures of adiposity, we hypothesised that that these same eating patterns would be related to (obesity-associated) BP and hypertension [20,23].

\section{Subjects and methods}

\section{Sample and study design}

This study is a secondary analysis of data drawn from the nationally representative, cross-sectional 2011-2012 Australian National Nutrition and Physical Activity Survey (NNPAS 2011-12) and was registered at anzctr.org.au as ACTRN12617001029381. The NNPAS, conducted between May 2011 and June 2012, was administered by the Australian Bureau of Statistics (ABS); full details of the study design and methods have been described elsewhere [25]. Briefly, 12,153 participants aged 2 or more years (including 9338 adults, aged $\geq 19$ years; $77 \%$ response rate) were selected using a multistage, probability sampling design of private dwellings (Fig. 1). The Australian Government Census and Statistics Act 1905 provided the ABS with ethics approval to conduct the survey components of the NNPAS [25].

\section{Blood pressure}

All BP measurements were voluntary, excluded pregnant women, and were taken by trained ABS staff during the home visit. Participants were asked to sit comfortably and relax their left arm. Two SBP and DBP measurements were taken on the left arm, with the palm facing upwards, using an automated BP monitor. The second reading was used, except where there was greater than $10 \mathrm{mmHg}$ difference between the first and second readings, in which case, a third reading was taken and the second and third readings averaged. If all three readings differed from each other by $>20 \mathrm{mmHg}$, then these readings were considered invalid. Participants were classified as hypertensive $(\geq 140 / 90 \mathrm{mmHg}$ ) or nonhypertensive $(<140 / 90 \mathrm{mmHg})$ [26]. No data about use of BP medications were collected; however, participants' selfreported whether they had current or previous hypertensive disease.

\section{Dietary assessment}

Dietary data were collected over two 24-h recalls, conducted approximately 9 days apart, on a different day of the week. During each dietary recall, based on the validated USDA automated multiple 5-pass method, participants were asked to identify the type of EO (e.g., breakfast, lunch, dinner, or snack) and the time when each EO commenced. The Australian Supplement and Nutrient Database 2011-2013 was used to determine energy and nutrient intakes from all foods and beverages and dietary information was averaged across the two recall days to obtain mean estimates of eating patterns, total energy intakes, and food intakes.

\section{Frequencies of all EO, meals, and snacks}

The methods used to calculate mean total frequencies of all EO, meals, and snacks have been described previously $[18,27]$. Briefly, an EO constituted any eating event that provided a minimum energy content of $210 \mathrm{~kJ}(50 \mathrm{kcal})$ and was separated in time from the surrounding EO by $15 \mathrm{~min}$. This approach was informed by the previous research and current recommendations for defining EO in eating patterns research $[4,27]$. EO were then classified as meals and snacks according to participants' self-report of EO. Meals included EO reported as breakfast, brunch, lunch, dinner, and supper, whereas snacks included EO reported as morning/afternoon tea and beverage break were classified as snack EO. Based on the sample distribution, frequencies of all EO, meals and snacks were divided into categories of $1-3,4-5$, or $\geq 6$ EO, $1-2,3$, or $>3$ meals, and $0-1,2-3$, or $>3$ snacks. 
Fig. 1 Flowchart of included participants from the 20112012 Australian National Nutrition and Physical Activity Survey (NNPAS 2011-12)

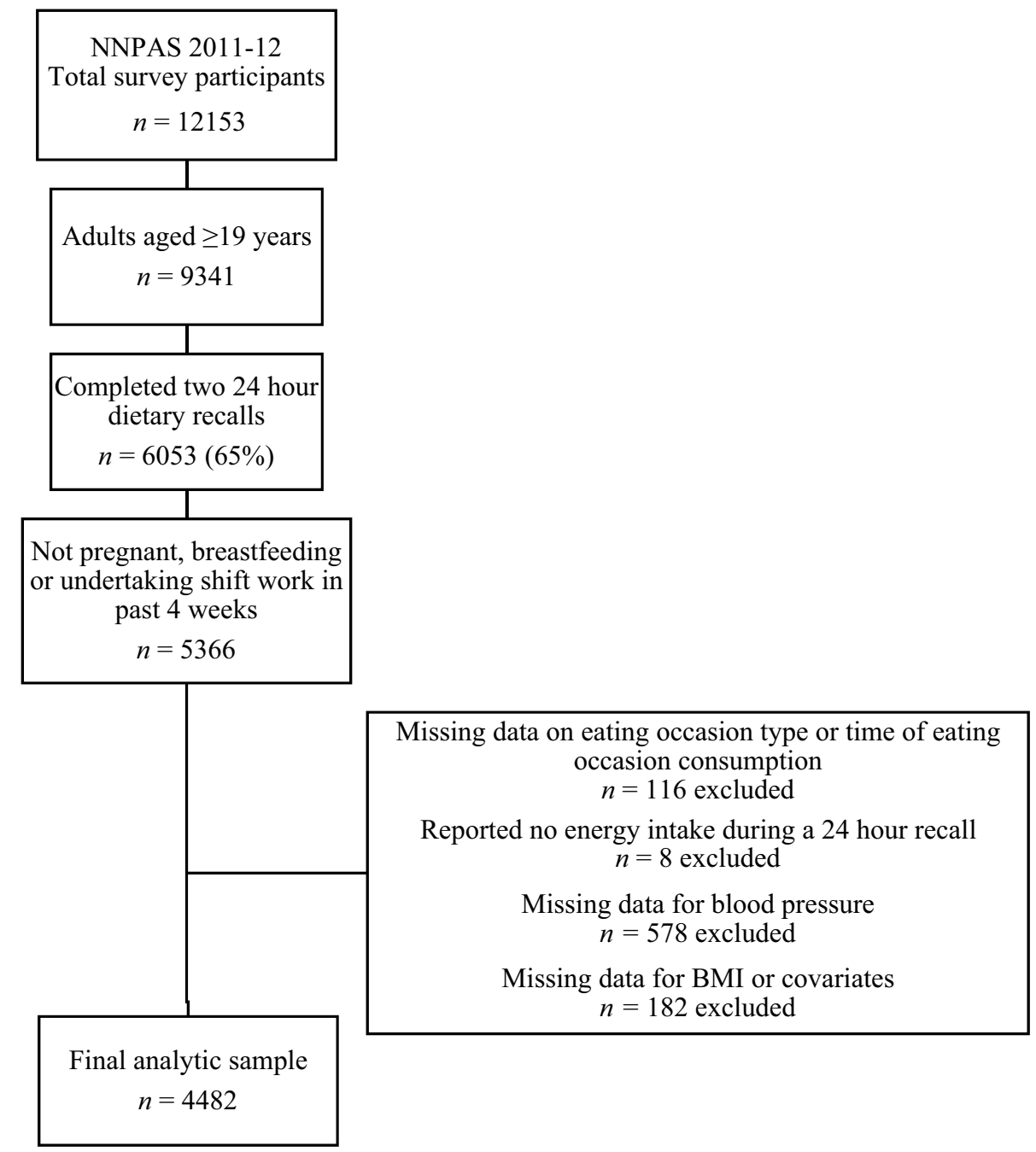

\section{Temporal eating patterns}

Temporal eating patterns were determined using latent class analysis, as reported in detail previously [23, 28]. Briefly, three latent classes of temporal eating patterns were identified for men and women based on frequency and timing of EO across the day and labelled according to their defining characteristics. The optimal number of classes was selected based on model fit indices, likelihood ratio tests comparing $k$ with $k^{-1}$ class models, and pattern interpretability [29]. The first pattern was labelled as "conventional" due to the probability of participants having three EO at "conventional" mealtimes in Australia (e.g., between 7 and 8 a.m., 12-1 p.m., and 6-7 p.m.). The second pattern was distinguished by a $>0.9$ probability of a "lunch" meal approximately $1 \mathrm{~h}$ later than the "conventional pattern" and labelled the "later lunch" pattern. The probability of a "dinner" meal $1 \mathrm{~h}$ later than the conventional pattern was also higher (e.g., $>0.6$ ) in participants with a "later lunch" pattern. The third pattern was labelled the "Grazing" pattern, because it was characterized by frequent but less distinct meal occasions and a higher probability of having an EO after 8 p.m. These temporal eating patterns have been associated with sociodemographic and eating pattern characteristics [28], diet quality, and, among women, adiposity outcomes [23].

\section{Covariates}

The following socio-demographic, health behaviours, and anthropometric variables, collected during the household survey, were considered as potential covariates due to their previous reported relation with hypertension risk $[1,26]$.

\section{Socio-demographics}

Education level was categorised as: low (completed some high-school or less), medium (completed high-school or completed some high-school and/or certificate/diploma) or high (having a tertiary qualification). Country of birth was categorised by the ABS as: Australia, predominantly 
English-speaking countries (other than Australia) and all other countries.

\section{Health behaviours}

Smoking status was self-reported and categorised as current smoker, ex-smoker, and never smoked. Participants were categorised as meeting or not meeting current Australian physical activity guidelines (150 min and 5 sessions), based on self-reported frequency and duration of walking for recreation or transport and moderate or vigorous leisuretime physical activity [30, 31]. Self-reported information on sleep duration the night before the survey and how much time participants spent sitting or lying down at work, during transport and leisure activities in the past week, were used to calculate (per day) total minutes spent sleeping per day and in sedentary behaviour, respectively. Participants reported whether they were currently on a weight-loss diet for health reasons (yes/no). Average daily total energy intake and diet quality scores were calculated from the 2 days of recall. The established food-based Dietary Guidelines Index (DGI) was used as a measure of overall diet quality [32-34]. The DGI assesses compliance with recommendations outlined in the Australian Dietary Guidelines, and is the sum of $13 \mathrm{com}-$ ponents (score range of 0-130), each corresponding to an Australian Dietary guideline and scored proportionally out of 10 . The components include meeting recommendations for food variety, intakes of fruits, vegetables (including legumes), grain foods, dairy and alternatives, meat and alternatives, unsaturated fat, fluids, discretionary foods, saturated fat, salt, added sugar, and alcohol. Higher scores indicate a better diet quality. Measurement of height $(\mathrm{cm})$ and weight $(\mathrm{kg})$ were taken to one decimal point by trained ABS staff using a portable stadiometer and digital scales. BMI (weight $[\mathrm{kg}] /$ height $[\mathrm{m}]^{2}$ ) was calculated.

\section{Analytic sample}

The analytic sample included the $65 \%$ of adult participants who completed both dietary recalls $(n=6053$; Fig. 1$)$. Participants were eligible for this analysis if they were not pregnant, breastfeeding, or undertaking shift-work in the past 4 weeks $(n=5366)$ and were excluded if they reported no energy intake during either dietary recall ( $n=8$ excluded) or did not report the time at which an EO commenced or the type of EO ( $n=116$ excluded). Of the remaining 5242 participants, 578 (11\%) had missing data for BP and a further 182 (3.9\%) were missing data for BMI and covariates: BMI $(n=149)$, physical activity $(n=28)$, and sedentary time $(n=5)$. The final analytic sample was 2099 men and 2383 women.

\section{Statistics}

All statistical analyses were stratified by sex and used Stata statistical software, Version 14.2 (Stata Inc., College Station, TX, USA). Point estimates and standard errors were determined by applying person and replicate weights that accounted for the probability of participant selection and the clustered survey design, respectively. Descriptive statistics for sample characteristics are presented as weighted means $(95 \% \mathrm{CI})$ or weighted percentages. After examining the distribution of the data, the following variables were log-transformed to improve normality: BMI, daily total sedentary time, and total energy intake. Weighted geometric means $(95 \% \mathrm{CI})$ were used for all log-transformed variables.

The $F$ test (for continuous data) and adjusted Pearson $\chi^{2}$ test (for categorical data) were used to determine sex-specific differences in sample characteristics by hypertension status. Multiple linear regression (for continuous outcomes) and logistic regression (for binary outcomes) were used to test for associations of frequencies of all EO, meal and snacks (continuous), and temporal eating patterns, with SBP and DBP (continuous) and hypertension prevalence (binary). Four models were tested: model 1 was an unadjusted model; model 2 adjusted for age (years, continuous), education level (low, medium or high), country of birth (Australia, other predominantly English-speaking countries, all other countries), smoking status (never, former or current), daily, meeting physical activity guidelines (yes/no), daily sedentary time (min; continuous), sleep duration ( $\mathrm{h}$, continuous), dieting for health reasons (yes/no); model 3 further adjusted for BMI, and model 4 further adjusted for total energy intake and DGI scores (both continuous). In light of the previous research that reported a positive association among participants with the highest EO frequencies (i.e., $>5 \mathrm{EO}$ ) [13], in the present study, any observed statistically significant $(P<0.05)$ adjusted association between the continuous measures of frequencies of EO, meals, or snacks, and the outcome variables were further explored by examining associations for eating pattern frequency categories (e.g., 1-3 [reference], $4-5$ or $\geq 6$ EO; $1-2$ [reference], 3 or $>3$ meals and $0-1$ [reference], and 2-3 or $>3$ snacks). Finally, the effect of energy misreporting, defined as the ratio of total energy intake to total energy expenditure was considered [35]; however, its inclusion did not improve its predictive power when BMI was already in the model. A previous study has also shown that energy misreporting bias can be statistically corrected using predictors of energy misreporting (i.e., dieting behaviours and BMI) [36].

\section{Sensitivity analysis}

As data on BP medications use were not collected in the NNPAS [25], a sensitivity analysis was conducted that 
included only participants who self-reported no current or previous hypertensive disease ( $n=1612$ men and $n=1853$ women).

\section{Results}

Table 1 presents the characteristics of men and women participants in the NNPAS 2011-12 by hypertension status. Of the participants, $24 \%$ of men and $20 \%$ of women were classified as having hypertension. Among both sexes, there were significant differences by hypertension status for age, education level, meeting physical activity guidelines, BMI, and self-report status of previous or current hypertensive disease $(P<0.05)$. Differences were also found for total daily energy intake, DGI scores, and frequency of all EO and snacks between non-hypertensive and hypertensive men $(P<0.05)$. For the snack frequency categories, a higher and lower proportion of hypertensive men reported having fewer than two snacks and more than three snacks per day, respectively, compared to non-hypertensives $(P<0.05)$. No significant differences were found among women for the dietary or eating pattern variables according to hypertension status.

The sex-specific associations of frequency of all EO, meals, and snacks (continuous) with SBP and DBP are presented in Table 2 . In the basic model, a statistically significant positive association was found between meal frequency and SBP among women which disappeared after adjustment for the covariates in model 2. Among men, inverse associations were found between frequency of all EO and snacks and DBP, and after adjustment for covariates (model 2) and BMI (model 3).

After further analysis that examined these associations by categories of snack and EO frequency, the inverse associations were observed among men who reported $>3$ snacks [DBP: $\beta=-2.23,95 \%$ CI $(-4.14,-0.32) ; P=0.023$ ] and $\geq 6$ EO [DBP: $\beta=-2.32,95 \%$ CI $(-4.45,-0.20)$; $P=0.032]$, compared to those who reporting $<2$ snacks and $\leq 3 \mathrm{EO}$. However, these associations with DBP for men were attenuated after further adjustment for the total energy intake and DGI scores: $\beta=-1.56,95 \% \mathrm{CI}(-3.59,0.47) ; P=0.13$ and $\beta=-1.37,95 \% \mathrm{CI}(-3.62,0.89) ; P=0.23$, respectively.

Results of the regression analyses showed no associations between latent classes of temporal eating patterns and SBP or DBP among men (Table 2). Among women, a "later lunch" temporal eating pattern was positively associated with SBP and DBP, when compared to a "conventional" pattern, after adjustment for covariates and BMI, and after further adjustment for dietary intakes.

Associations of frequency of all EO, meals, and snacks (continuous) and latent classes of temporal eating patterns with hypertension prevalence are shown in Table 3. Among men, a significant inverse association was found for frequency of all EO and snacks but not meals.

After further analysis that examined these associations by categories of snack and EO frequency, the inverse adjusted associations (model 3) were only observed among men who reported $>3$ snacks [OR $0.52,95 \%$ CI $(0.30,0.81)$; $P=0.006$ ] and $\geq 6$ EO [OR $0.54,95 \%$ CI $(0.34,0.84)$; $P=0.008]$, compared to those who reporting $<2$ snacks and $\leq 3 \mathrm{EO}$. However, again, these associations attenuated after further adjustment for total energy intake and DGI scores, and no significant associations were found among women. Compared to a "conventional" temporal eating pattern, a "later lunch temporal" eating pattern was also associated with hypertension prevalence among women, but only after adjustment for covariates and BMI scores.

Results of the sensitivity analyses which included only men and women with no self-reported current or previous hypertensive disease, showed similar null associations of frequency of EO, snacks, and meals with SBP, DBP or hypertension prevalence, after adjustment for covariates, BMI, and dietary intakes (Supplementary Table 1). However, among women, the finding of a positive association between a "later lunch" pattern and DBP (but not SBP or hypertension prevalence) persisted after exclusion of those with no self-reported current/previous hypertensive disease.

\section{Discussion}

To our knowledge, this is one of the first studies among adults to examine associations of meal and snack frequency and temporal eating patterns, based on the timing and frequency of EO across the day, with BP and hypertension prevalence [12]. Among men, frequency of all EO and snacks was inversely associated with DBP and lower odds of hypertension prevalence, but these associations disappeared after adjustment for overall diet quality scores and total energy intakes. Among women, a "later lunch" pattern, identified using latent class analysis in our earlier study [28], and characterized by a later lunch EO (e.g., between 1 and 2 p.m.) was associated with higher SBP, DBP, and hypertension prevalence. However, only associations with DBP persisted after exclusion of persons with the self-reported previous/current hypertension.

Only a few studies have examined the relationship between EO frequency and BP among adults $[12,13,16$, 17], with conflicting findings. However, it is difficult to compare the results of these studies, because they define EO using different approaches. For example, EO have mostly been self-reported by participants in response to a single survey question where an EO is not further defined [12, 17]. Whereas in another study, in a small sample of healthy volunteers $(n=115)$, EO was defined as any eating event 
Table 1 Characteristics of men and women in the NNPAS by hypertension status

\begin{tabular}{|c|c|c|c|c|c|c|}
\hline & \multicolumn{3}{|l|}{$\operatorname{Men}(n=2099)$} & \multicolumn{3}{|l|}{ Women $(n=2383)$} \\
\hline & $\begin{array}{l}\text { Non-hypertensive } \\
(n=1538)\end{array}$ & Hypertensive $(n=561)$ & $P$ value & $\begin{array}{l}\text { Non-hypertensive } \\
(n=1883)\end{array}$ & Hypertensive $(n=500)$ & $P$ value \\
\hline \multicolumn{7}{|l|}{ Socio-demographics } \\
\hline Age (years) & $43.1(42.4,44.8)$ & $56.2(54.2,58.2)$ & $<0.0001$ & $44.7(44.0,45.5)$ & $58.4(56.8,60.0)$ & $<0.0001$ \\
\hline Education level (\%) & & & $<0.05$ & & & $<0.0001$ \\
\hline Low & 18 & 25 & & 25 & 43 & \\
\hline Medium & 53 & 54 & & 44 & 33 & \\
\hline High & 29 & 21 & & 32 & 24 & \\
\hline Country of birth (\%) & & & 0.95 & & & 0.12 \\
\hline Australia & 69 & 69 & & 70 & 61 & \\
\hline $\begin{array}{l}\text { Predominantly } \\
\text { English-speaking } \\
\text { countries }\end{array}$ & 13 & 13 & & 11 & 15 & \\
\hline All other countries & 18 & 18 & & 19 & 24 & \\
\hline \multicolumn{7}{|l|}{$\begin{array}{l}\text { Health behaviours or } \\
\text { characteristics }\end{array}$} \\
\hline Smoking status & & & 0.14 & & & 0.56 \\
\hline Never & 47 & 39 & & 59 & 55 & \\
\hline Former & 35 & 40 & & 27 & 31 & \\
\hline Current & 18 & 20 & & 14 & 14 & \\
\hline $\begin{array}{l}\text { Meets physical activ- } \\
\text { ity guidelines }{ }^{\mathrm{a}}(\%)\end{array}$ & 48 & 41 & 0.04 & 45 & 35 & $<0.01$ \\
\hline $\begin{array}{l}\text { Daily sedentary time } \\
\text { (min) }\end{array}$ & $306.7(289.8,324.6)$ & $297.2(273.0,323.4)$ & 0.54 & $260.3(247.9,273.2)$ & $263.1(237.0,292.1)$ & 0.86 \\
\hline $\begin{array}{l}\text { Currently on a diet } \\
\text { for health reasons } \\
(\%)\end{array}$ & 12 & 8 & 0.06 & 17 & 15 & 0.43 \\
\hline Sleep duration (h) & $7.9(7.8,8.0)$ & $7.9(7.7,8.0)$ & 0.81 & $8.0(7.9,8.1)$ & $8.0(7.7,8.2)$ & 0.52 \\
\hline $\begin{array}{l}\text { Total energy intake } \\
(\mathrm{kJ})\end{array}$ & $9498(9304,9696)$ & $8463(8170,8766)$ & $<0.0001$ & $7087(6928,7250)$ & $6890(6587,7206)$ & 0.31 \\
\hline $\begin{array}{l}\text { Dietary Guidelines } \\
\text { Index }^{\mathrm{c}} \text { (score) }\end{array}$ & $80.1(79.0,81.2)$ & $77.6(75.7,79.6)$ & $<0.05$ & $80.7(79.5,82.0)$ & $81.8(80.1,83.5)$ & 0.31 \\
\hline $\mathrm{BMI}^{\mathrm{b}}$ (score) & $26.8(26.5,27.2)$ & $28.9(28.2,29.5)$ & $<0.0001$ & $25.9(25.5,26.3)$ & $28.7(27.9,29.6)$ & $<0.0001$ \\
\hline $\begin{array}{l}\text { Systolic blood pres- } \\
\text { sure }(\mathrm{mmHg})\end{array}$ & $118.7(117.9,119.5)$ & $148.3(145.6,151.0)$ & $<0.0001$ & $112.5(111.7,113.1)$ & $148.8(146.9,150.8)$ & $<0.0001$ \\
\hline $\begin{array}{l}\text { Diastolic blood pres- } \\
\text { sure }(\mathrm{mmHg})\end{array}$ & $73.4(72.8,74.0)$ & $88.7(87.5,89.8)$ & $<0.0001$ & $72.9(72.2,73.6)$ & $89.0(87.9,90.2)$ & $<0.0001$ \\
\hline $\begin{array}{l}\text { No current or previ- } \\
\text { ous hypertensive } \\
\text { disease }(\%)\end{array}$ & 85 & 63 & $<0.0001$ & 86 & 56 & $<0.0001$ \\
\hline \multicolumn{7}{|l|}{ Eating patterns } \\
\hline $\begin{array}{l}\text { Eating occasion } \\
\text { frequency }\end{array}$ & $4.9(4.8,5.0)$ & $4.7(4.5,4.8)$ & $<0.01$ & $4.8(4.7,4.9)$ & $4.7(4.6,4.9)$ & 0.37 \\
\hline Meal frequency & $2.9(2.8,2.9)$ & $2.9(2.8,2.9)$ & 0.79 & $2.9(2.9,3.0)$ & $3.0(2.9,3.0)$ & 0.55 \\
\hline Snack frequency & $2.1(1.98,2.2)$ & $1.8(1.7,2.0)$ & $<0.01$ & $1.9(1.9,2.0)$ & $1.8(1.7,2.0)$ & 0.19 \\
\hline $\begin{array}{l}\text { Categories of eating } \\
\text { occasion frequency } \\
(\%)\end{array}$ & & & 0.07 & & & 0.54 \\
\hline $1-3$ & 19 & 24 & & 18 & 20 & \\
\hline $4-5$ & 57 & 60 & & 61 & 62 & \\
\hline$\geq 6$ & 23 & 17 & & 21 & 18 & \\
\hline $\begin{array}{l}\text { Categories of meal } \\
\text { frequency }(\%)\end{array}$ & & & 0.27 & & & 0.89 \\
\hline
\end{tabular}


Table 1 (continued)

\begin{tabular}{|c|c|c|c|c|c|c|}
\hline & \multicolumn{3}{|l|}{$\operatorname{Men}(n=2099)$} & \multicolumn{3}{|l|}{ Women $(n=2383)$} \\
\hline & $\begin{array}{l}\text { Non-hypertensive } \\
(n=1538)\end{array}$ & Hypertensive $(n=561)$ & $P$ value & $\begin{array}{l}\text { Non-hypertensive } \\
(n=1883)\end{array}$ & Hypertensive $(n=500)$ & $P$ value \\
\hline$<3$ & 31 & 32 & & 26 & 25 & \\
\hline 3 & 53 & 48 & & 54 & 54 & \\
\hline$>3$ & 15 & 20 & & 20 & 21 & \\
\hline $\begin{array}{l}\text { Categories of snack } \\
\text { frequency }(\%)\end{array}$ & & & $<0.05$ & & & 0.59 \\
\hline$<2$ & 45 & 51 & & 48 & 51 & \\
\hline $2-3$ & 37 & 38 & & 39 & 38 & \\
\hline$>3$ & 18 & 11 & & 13 & 11 & \\
\hline $\begin{array}{l}\text { Latent classes of } \\
\text { temporal eating pat- } \\
\text { terns }(\%)\end{array}$ & & & 0.09 & & & 0.17 \\
\hline Conventional & 40 & 47 & & 41 & 37 & \\
\hline Later lunch & 36 & 30 & & 32 & 39 & \\
\hline Grazing & 24 & 23 & & 27 & 24 & \\
\hline
\end{tabular}

Values are weighted means (95\% confidence intervals) or weighted percentages. Significant sex-specific differences by hypertension status assessed using an $F$ test for continuous variables or design-adjusted Pearson $\chi^{2}$ test

${ }^{a}$ Whether met physical activity guidelines of $150 \mathrm{~min}$ and 5 sessions/week

${ }^{\mathrm{b}}$ Values are geometric means $(95 \% \mathrm{CI})$

${ }^{\mathrm{c}}$ DGI represents a total diet quality score (score range 0-130) with higher scores indicating better overall diet quality

(including kilojoule-free events) separated in time by $15 \mathrm{~min}$ [13]. A higher EO frequency has been associated with lower hypertension prevalence [12] and incidence [13], whereas other studies have found no associations with BP [16, 17]. In the present study, EO frequency was inversely associated with DBP and hypertension prevalence among men, but these associations attenuated after further adjustment for total energy intakes and overall diet quality.

Studies examining the separate effects of meal and snack frequency on BP outcomes are rare [12]. Compared to participants who reported no snacks, Kim et al. [12] found that a snack frequency of three per day was associated with lower odds of hypertension, but associations attenuated (e.g., 95\% $\mathrm{CI}$ included one) after adjustment for adiposity measures. In the present study, snack frequency (specifically $>3$ snacks) was inversely associated with BP outcomes among men, but again associations attenuated after adjustment for overall diet quality and total energy intakes. Notably, with respect to adjustment of dietary factors, the previous studies on eating patterns and BP have only adjusted for either total energy intakes [13] or energy and nutrient intakes [12, 16, 17], and not a measure of overall diet quality based on food intakes.

The findings from the present study suggest that diet quality may be an important factor in the relation between EO frequency and BP, and is supported by the previous studies that have shown a beneficial effect of healthful dietary patterns for the prevention of hypertension [37]. In a previous study of NNPAS participants. [18], snack frequency was associated with better diet quality scores for intakes of fruits and dairy products, two food groups recommended as part of the Dietary Approaches to Stop Hypertension (DASH) diet [3]. However, in the same study, snack frequency was also associated with poorer scores for intakes of discretionary foods and added sugars among men [18]. Future research that examines the role of diet quality on the relation between EO frequency and hypertension is warranted.

Epidemiological evidence suggests a positive association between evening energy intakes or the later timing of an EO and obesity [38], but studies examining temporal patterns of eating in relation to BP are rare. In one study, higher energy intake at breakfast was associated with lower hypertension prevalence but not 10-year incidence, and higher energy intake in the evening was associated with higher hypertension incidence, which remained borderline significant after adjustment for baseline BMI [21]. Another study, in the same cohort, found no association between time of day macronutrient intakes and BP [39]. Keller et al. found that the consumption of an afternoon meal, but not other conventional Spanish meals, was modestly associated with lower SBP and DBP, even after adjustment for dietary intake and waist circumference [22]. In the present study, a temporal eating pattern characterized by having a later "lunch" meal was associated with SBP, DBP, and hypertension prevalence among women, after adjustment for potential covariates, BMI, and diet quality. However, rather than examining the timing of a single meal or energy intake across stratified 
Table 2 Associations of eating patterns with systolic and diastolic blood pressure in Australian men and women

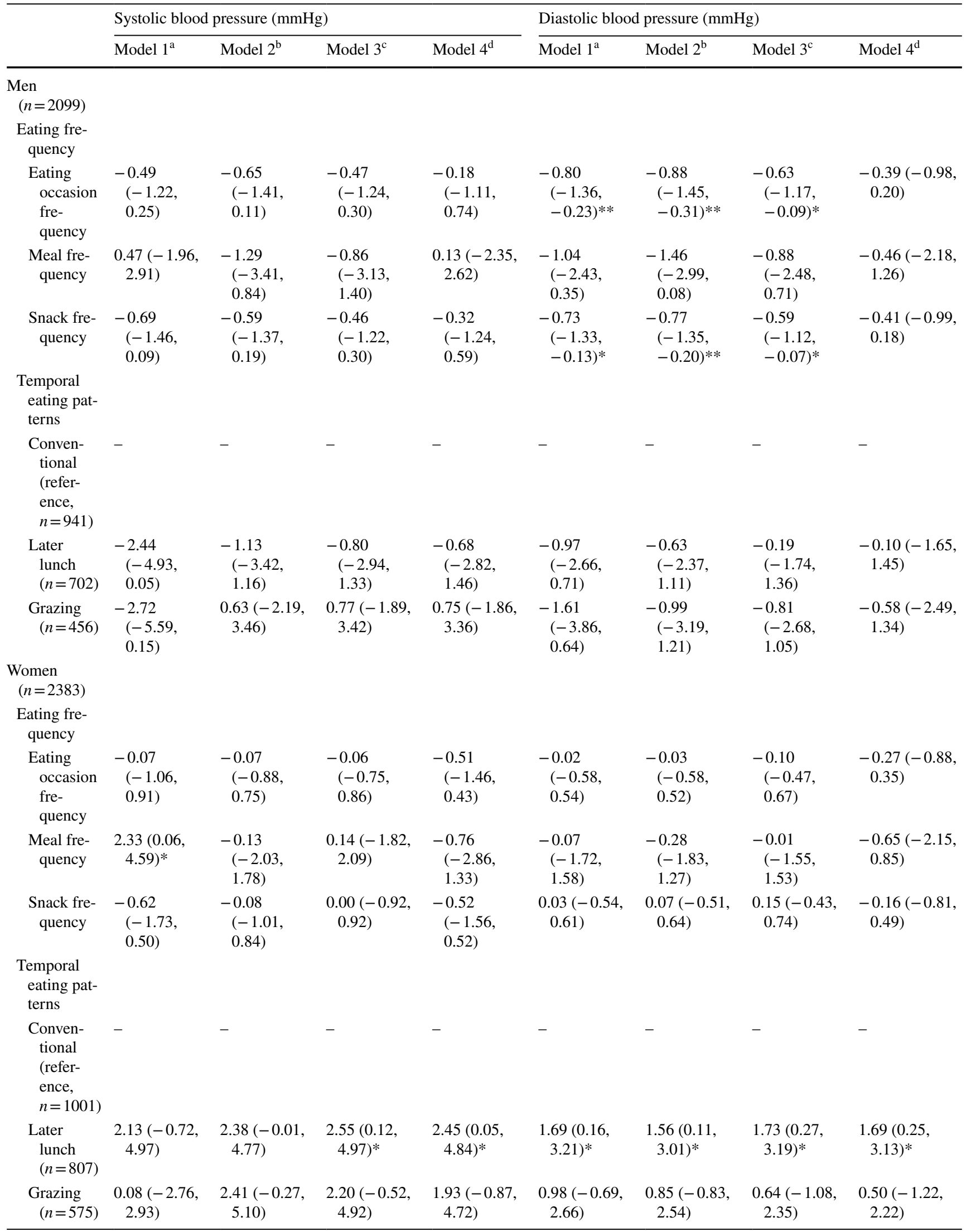


Table 2 (continued)

Values are presented as $\beta$ coefficients (95\% confidence intervals). Associations were examined using the Wald tests of associations for linear regression; $* P<0.05, * * P<0.01$

${ }^{\mathrm{a}}$ Crude analysis

${ }^{b}$ Adjusted for age (years, continuous), sedentary time (min/day, continuous), education level (low/medium/high), country of birth (Australia/ other mainly English-speaking countries/all other countries), meets PA guidelines (yes/no), smoking status (never smoked/past smoker/current smoker), and dieting (yes/no)

${ }^{\mathrm{c}}$ Model 2 and additionally adjusted for BMI scores

${ }^{\mathrm{d}}$ Model 3 and additionally adjusted for Dietary Guideline Index scores and total energy intake

Table 3 Associations of eating patterns with hypertension prevalence in Australian men and women

\begin{tabular}{|c|c|c|c|c|}
\hline & Model $1^{\mathrm{a}}$ & Model $2^{\mathrm{b}}$ & Model $3^{\mathrm{c}}$ & Model $4^{\mathrm{d}}$ \\
\hline \multicolumn{5}{|l|}{ Men $(n=2099)$} \\
\hline \multicolumn{5}{|l|}{ Eating frequency } \\
\hline Eating occasion frequency & $0.87(0.80,0.97)^{*}$ & $0.85(0.75,0.96)^{* *}$ & $0.87(0.76,0.98)^{*}$ & $0.94(0.81,1.08)$ \\
\hline Meal frequency & $1.03(0.76,1.40)$ & $0.88(0.64,1.21)$ & $0.92(0.66,1.30)$ & $1.12(0.77,1.61)$ \\
\hline Snack frequency & $0.86(0.76,0.96)^{*}$ & $0.85(0.75,0.96)^{*}$ & $0.86(0.75,0.98)^{*}$ & $0.91(0.79,1.05)$ \\
\hline \multicolumn{5}{|l|}{ Temporal eating patterns } \\
\hline Conventional (reference, $n=941$ ) & - & - & - & - \\
\hline Later lunch $(n=702)$ & $0.70(0.52,0.93)^{*}$ & $0.78(0.56,1.08)$ & $0.81(0.59,1.12)$ & $0.82(0.59,1.13)$ \\
\hline Grazing $(n=456)$ & $0.81(0.55,1.20)$ & $1.14(0.74,1.74)$ & $1.15(0.76,1.75)$ & $1.22(0.81,1.82)$ \\
\hline \multicolumn{5}{|l|}{ Women $(n=2383)$} \\
\hline \multicolumn{5}{|l|}{ Eating frequency } \\
\hline Eating occasion frequency & $0.94(0.83,1.08)$ & $0.95(0.83,1.08)$ & $0.97(0.84,1.10)$ & $0.93(0.79,1.09)$ \\
\hline Meal frequency & $1.10(0.81,1.50)$ & $0.94(0.70,1.27)$ & $0.96(0.70,1.31)$ & $0.93(0.79,1.09)$ \\
\hline Snack frequency & $0.91(0.79,1.05)$ & $0.95(0.83,1.09)$ & $0.95(0.82,1.10)$ & $0.93(0.79,1.09)$ \\
\hline \multicolumn{5}{|l|}{ Temporal eating patterns } \\
\hline Conventional (reference, $n=1001$ ) & - & - & - & - \\
\hline Later lunch $(n=807)$ & $1.34(0.94,1.90)$ & $1.47(1.00,2.16)$ & $1.51(1.01,2.25)^{*}$ & $1.49(1.00,2.22) *$ \\
\hline Grazing $(n=575)$ & $0.97(0.64,1.45)$ & $1.11(0.71,1.73)$ & $1.06(0.67,1.68)$ & $1.04(0.64,1.67)$ \\
\hline
\end{tabular}

Values are presented as odds ratios (95\% confidence intervals). Associations were examined using the Wald tests of associations for logistic regression; $* P<0.05, * * P<0.01$

${ }^{\mathrm{a}}$ Crude analysis

${ }^{b}$ Adjusted for age (years, continuous), sedentary time (min/day, continuous), education level (low/medium/high), country of birth (Australia/ other mainly English-speaking countries/all other countries), meets PA guidelines (yes/no), smoking status (never smoked/past smoker/current smoker), and dieting (yes/no)

${ }^{\mathrm{c}}$ Model 2 and additionally adjusted for BMI scores

${ }^{\mathrm{d}}$ Model 3 and additionally adjusted for Dietary Guideline Index scores and total energy intake

time-periods, the present study examined temporal eating patterns based on a novel latent class analysis approach, which captures the timing multiple EO across the day and the likely correlations of energy intakes between EO [4].

The possible mechanisms by which the later timing of a "lunch" meal might increase SBP and DBP among women are unclear. The timing of the "dinner" (evening) meal also tended to be later in women with this pattern and research has shown that insulin sensitivity gradually lowers across the day, into the evening [40]. In addition, in an experimental trial that controlled for dietary intakes, the timing of an evening meal high in energy and carbohydrates but low in fibre was associated with reduced insulin sensitivity and higher blood glucose levels, when compared to participants who had the same meal in the morning [41]. Insulin metabolism may contribute to the association between temporal eating patterns and BP due to its role in modulating vasodilator effects on the endothelium via nitric oxide bioavailability [40]. Measures of insulin sensitivity and blood glucose levels in future epidemiological research examining temporal eating patterns and BP are needed.

Strengths of this study include the examination of associations with BP in a large nationally representative sample, adjusted for BMI, and multiple important confounders, including a measure of overall diet quality. Eating patterns were determined from 2 days of dietary recall, and an EO was 
defined using an evidence-based approach [27]. While the novel methodology used to determine temporal eating patterns is also considered a study strength, it should be noted that findings from data-driven, exploratory methods may not be generalizable to populations from other countries. For example, the temporal eating patterns found in the present study may reflect the sociocultural and lifestyle characteristics of the Australian adult population; further research is needed to better understand differences in temporal eating patterns in other populations. Limitations of the present study include the cross-sectional study design which does not permit the assessment of temporal relationships and the lack of data on antihypertensive medications which may have led to an underestimation of associations with BP. However, in the analysis that excluded participants with no current/previous self-reported hypertension, the association between a "later lunch pattern" with DBP persisted.

In conclusion, a temporal eating pattern distinguished by a later "lunch" meal when compared to a "conventional" Australian mealtime pattern was associated with higher BP in women. Among men, a higher snack frequency was inversely associated with BP, but these associations disappeared after adjustment for total energy intake and overall diet quality. Future research that considers the role of overall diet quality and cardiometabolic indicators (e.g. insulin and glucose levels) on the relation between eating patterns and $\mathrm{BP}$ is warranted.

Acknowledgements A.T. was supported by a National Heart Foundation of Australia Future Leader Fellowship (Award 100046). S.A.M. is supported by a National Health and Medical Research Council Career Development Fellowship (ID1104636). R.M.L. is supported by an Alfred Deakin Postdoctoral Research Fellowship. The authors would like to acknowledge Dr. Katherine Livingstone for the data preparation of the diet quality score variable used in this study.

\section{Compliance with ethical standards}

Research involving human participants and/or animals This research does not include patient data or data from a clinical trial. This research is secondary analysis of Australian (national) Health Survey (AHS) data commissioned by the Commonwealth of Australia and conducted by the Australian Bureau of Statistics (ABS). This secondary analysis was registered at anzctr.org.au as ACTRN12617001029381. The web address of this trial is: http://www.ANZCTR.org.au/ACTRN12617 001029381.aspx. Ethical approval for the ABS to conduct the household components of the AHS is provided by the Australian Federal Government Census and Statistics Act, 1905 (https://www.legislatio n.gov.au/Details/C2006C00178).

Conflict of interest On behalf of all authors, the corresponding author states that there is no conflict of interest.

Open Access This article is distributed under the terms of the Creative Commons Attribution 4.0 International License (http://creativeco mmons.org/licenses/by/4.0/), which permits unrestricted use, distribution, and reproduction in any medium, provided you give appropriate credit to the original author(s) and the source, provide a link to the Creative Commons license, and indicate if changes were made.

\section{References}

1. Collaborators GBDRF. (2017) Global, regional, and national comparative risk assessment of 84 behavioural, environmental and occupational, and metabolic risks or clusters of risks, 1990-2016: a systematic analysis for the Global Burden of Disease Study 2016. Lancet 390(10100):1345-1422. https://doi.org/10.1016/ S0140-6736(17)32366-8

2. Estruch R, Ros E, Martinez-Gonzalez MA (2013) Mediterranean diet for primary prevention of cardiovascular disease. N Engl J Med 369(7):676-677. https://doi.org/10.1056/NEJMc1306659

3. Appel LJ, Brands MW, Daniels SR, Karanja N, Elmer PJ, Sacks FM, American Heart A (2006) Dietary approaches to prevent and treat hypertension: a scientific statement from the American Heart Association. Hypertension 47(2):296-308. https://doi. org/10.1161/01.HYP.0000202568.01167.B6

4. St-Onge MP, Ard J, Baskin ML, Chiuve SE, Johnson HM, KrisEtherton P, Varady K (2017) Meal timing and frequency: implications for cardiovascular disease prevention: a scientific statement from the American Heart Association. Circulation 135(9):e96e121. https://doi.org/10.1161/CIR.0000000000000476

5. Leech RM, Worsley A, Timperio A, McNaughton SA (2015) Understanding meal patterns: definitions, methodology and impact on nutrient intake and diet quality. Nutr Res Rev 28(1):121. https://doi.org/10.1017/S0954422414000262

6. Asher G, Sassone-Corsi P (2015) Time for food: the intimate interplay between nutrition, metabolism, and the circadian clock. Cell 161(1):84-92. https://doi.org/10.1016/j.cell.2015.03.015

7. Odegaard AO, Jacobs DR Jr, Steffen LM, Van Horn L, Ludwig DS, Pereira MA (2013) Breakfast frequency and development of metabolic risk. Diabetes Care 36(10):3100-3106. https://doi. org/10.2337/dc13-0316

8. Mekary RA, Giovannucci E, Cahill L, Willett WC, van Dam RM, $\mathrm{Hu}$ FB (2013) Eating patterns and type 2 diabetes risk in older women: breakfast consumption and eating frequency. Am J Clin Nutr 98(2):436-443. https://doi.org/10.3945/ajcn.112.057521

9. Pot GK, Almoosawi S, Stephen AM (2016) Meal irregularity and cardiometabolic consequences: results from observational and intervention studies. Proc Nutr Soc 75(4):475-486. https://doi. org/10.1017/S0029665116000239

10. Deshmukh-Taskar P, Nicklas TA, Radcliffe JD, O'Neil CE, Liu Y (2013) The relationship of breakfast skipping and type of breakfast consumed with overweight/obesity, abdominal obesity, other cardiometabolic risk factors and the metabolic syndrome in young adults. The National Health and Nutrition Examination Survey (NHANES): 1999-2006. Public Health Nutr 16(11):2073-2082. https://doi.org/10.1017/S1368980012004296

11. Sierra-Johnson J, Unden AL, Linestrand M, Rosell M, Sjogren P, Kolak M, De Faire U, Fisher RM, Hellenius ML (2008) Eating meals irregularly: a novel environmental risk factor for the metabolic syndrome. Obesity (Silver Spring) 16(6):1302-1307. https ://doi.org/10.1038/oby.2008.203

12. Kim S, Park GH, Yang JH, Chun SH, Yoon HJ, Park MS (2014) Eating frequency is inversely associated with blood pressure and hypertension in Korean adults: analysis of the Third Korean National Health and Nutrition Examination Survey. Eur J Clin Nutr. https://doi.org/10.1038/ejen.2014.9

13. Karatzi K, Georgiopoulos G, Yannakoulia M, Efthimiou E, Voidonikola P, Mitrakou A, Manios E, Alevizaki M, Papamichael C, Stamatelopoulos K (2016) Eating frequency predicts new onset hypertension and the rate of progression of blood pressure, arterial stiffness, and wave reflections. J Hypertens. https://doi. org/10.1097/hjh.0000000000000822

14. Karatzi K, Yannakoulia M, Psaltopoulou T, Voidonikola P, Kollias G, Sergentanis TN, Retsas T, Alevizaki M, Papamichael C, 
Stamatelopoulos K (2014) Meal patterns in healthy adults: inverse association of eating frequency with subclinical atherosclerosis indexes. Clin Nutr. https://doi.org/10.1016/j.clnu.2014.04.022

15. Bhutani S, Varady KA (2009) Nibbling versus feasting: which meal pattern is better for heart disease prevention? Nutr Rev 67(10):591-598. https://doi.org/10.1111/j.1753-4887.2009.00231 .x

16. Titan SM, Bingham S, Welch A, Luben R, Oakes S, Day N, Khaw KT (2001) Frequency of eating and concentrations of serum cholesterol in the Norfolk population of the European prospective investigation into cancer (EPIC-Norfolk): cross sectional study. BMJ 323(7324):1286-1288

17. Edelstein SL, Barrett-Connor EL, Wingard DL, Cohn BA (1992) Increased meal frequency associated with decreased cholesterol concentrations; Rancho Bernardo, CA, 1984-1987. Am J Clin Nutr 55(3):664-669

18. Leech RM, Livingstone KM, Worsley A, Timperio A, McNaughton SA (2016) Meal frequency but not snack frequency is associated with micronutrient intakes and overall diet quality in Australian men and women. J Nutr 146(10):2027-2034. https ://doi.org/10.3945/jn.116.234070

19. Murakami K, Livingstone MB (2015) Associations between meal and snack frequency and diet quality and adiposity measures in British adults: findings from the National Diet and Nutrition Survey. Public Health Nutr 19(9):1624-1634. https://doi.org/10.1017/ S1368980015002979

20. Leech RM, Worsley A, Timperio A, McNaughton SA (2017) The role of energy intake and energy misreporting in the associations between eating patterns and adiposity. Eur J Clin Nutr. https://doi. org/10.1038/ejen.2017.90

21. Almoosawi S, Prynne CJ, Hardy R, Stephen AM (2013) Timeof-day of energy intake: association with hypertension and blood pressure 10 years later in the 1946 British Birth Cohort. J Hypertens 31(5):882-892

22. Keller K, Rodriguez Lopez S, Carmenate Moreno M (2017) Association between meal intake behavior and blood pressure in Spanish adults. Nutr Hosp 34(3):654-660. https://doi.org/10.20960/ nh. 487

23. Leech RM, Timperio A, Livingstone KM, Worsley A, McNaughton SA (2017) Temporal eating patterns: associations with nutrient intakes, diet quality, and measures of adiposity. Am J Clin Nutr 106(4):1121-1130. https://doi.org/10.3945/ ajcn.117.156588

24. Eicher-Miller HA, Khanna N, Boushey CJ, Gelfand SB, Delp EJ (2016) Temporal dietary patterns derived among the adult participants of the National Health and Nutrition Examination Survey 1999-2004 are associated with diet quality. J Acad Nutr Diet 116(2):283-291. https://doi.org/10.1016/j.jand.2015.05.014

25. Australian Bureau of Statistics (2013) Australian health survey: users' guide, 2011-2013. Cat. no. 4363.0.55.001. ABS, Canberra

26. Chobanian AV, Bakris GL, Black HR, Cushman WC, Green LA, Izzo JL Jr, Jones DW, Materson BJ, Oparil S, Wright JT Jr, Roccella EJ, National Heart, Lung, and Blood Institute Joint National Committee on Prevention, Detection, Evaluation, and Treatment of High Blood Pressure, National High Blood Pressure Education Program Coordinating Committee (2003) The seventh report of the Joint National Committee on Prevention, Detection, Evaluation, and Treatment of High Blood Pressure: the JNC 7 report. JAMA 289 (19):2560-2572. https://doi.org/10.1001/ jama.289.19.2560

27. Leech RM, Worsley A, Timperio A, McNaughton SA (2015) Characterizing eating patterns: a comparison of eating occasion definitions. Am J Clin Nutr 102(5):1229-1237. https://doi. org/10.3945/ajen.115.114660

28. Leech RM, Worsley A, Timperio A, McNaughton SA (2017) Temporal eating patterns: a latent class analysis approach. Int J Behav Nutr Phys Act 14(1):3. https://doi.org/10.1186/s1296 6-016-0459-6

29. Lo Y, Mendell NR, Rubin DB (2001) Testing the number of components in a normal mixture. Biometrika 88(3):767-778. https:// doi.org/10.1093/biomet/88.3.767

30. Australian Institute of Health and Welfare (2003) The Active Australia Survey: a guide and manual for implementation, analysis and reporting. Cat. no. CVD 22. AIHW, Canberra

31. The Department of Health (2014) Australia's physical activity and sedentary behaviour guidelines [Webpage on the Internet]. DoH, Canberra (cited 26 Nov 2017)

32. National Health and Medical Research Council (2013) Australian dietary guidelines. NHMRC, Canberra

33. Thorpe MG, Milte CM, Crawford D, McNaughton SA (2016) A revised Australian dietary guideline index and its association with key sociodemographic factors, health behaviors and body mass index in peri-retirement aged adults. Nutrients 8(3):160. https:// doi.org/10.3390/nu8030160

34. Livingstone KM, McNaughton SA (2016) Diet quality is associated with obesity and hypertension in Australian adults: a cross sectional study. BMC Public Health 16(1):1037. https://doi. org/10.1186/s12889-016-3714-5

35. Banna JC, McCrory MA, Fialkowski MK, Boushey C (2017) Examining plausibility of self-reported energy intake data: considerations for method selection. Front Nutr 4:45. https://doi. org/10.3389/fnut.2017.00045

36. Vainik U, Konstabel K, Latt E, Maestu J, Purge P, Jurimae J (2016) Diet misreporting can be corrected: confirmation of the association between energy intake and fat-free mass in adolescents. Br J Nutr 116(8):1425-1436. https://doi.org/10.1017/s0007 114516003317

37. Folsom AR, Parker ED, Harnack LJ (2007) Degree of concordance with DASH diet guidelines and incidence of hypertension and fatal cardiovascular disease. Am J Hypertens 20(3):225-232. https://doi.org/10.1016/j.amjhyper.2006.09.003

38. Almoosawi S, Vingeliene S, Karagounis LG, Pot GK (2016) Chrono-nutrition: a review of current evidence from observational studies on global trends in time-of-day of energy intake and its association with obesity. Proc Nutr Soc 75(4):487-500. https:// doi.org/10.1017/S0029665116000306

39. Almoosawi S, Prynne CJ, Hardy R, Stephen AM (2013) Timeof-day and nutrient composition of eating occasions: prospective association with the metabolic syndrome in the 1946 British birth cohort. Int J Obes 37(5):725-731

40. Steinberg HO, Baron AD (2002) Vascular function, insulin resistance and fatty acids. Diabetologia 45(5):623-634. https://doi. org/10.1007/s00125-002-0800-2

41. Morgan LM, Shi J-W, Hampton SM, Frost G (2012) Effect of meal timing and glycaemic index on glucose control and insulin secretion in healthy volunteers. Br J Nutr 108(7):1286-1291 